\title{
Alteração no método centroide de avaliação da adaptabilidade genotípica
}

\author{
Moysés Nascimento(1), Cosme Damião Cruz(2), Ana Carolina Mota Campana(1), Rafael Simões Tomaz ${ }^{(2)}$, \\ Caio Césio Salgado(2) e Reinaldo de Paula Ferreira ${ }^{(3)}$
}

\begin{abstract}
(1)Universidade Federal de Viçosa (UFV), Departamento de Informática, Área de Estatística, Avenida P.H. Rolfs, s/no, CEP 36571-000 Viçosa, MG. E-mail: moysesnascim@vicosa.ufv.br, carolcampana@vicosa.ufv.br (2)UFV, Departamento de Biologia Geral. E-mail: cdcruz@ufv.br, rafaelstmz@gmail.com, caiocesio@yahoo.com.br (3)Embrapa Pecuária Sudeste, Rodovia Washington Luiz, Km 234, CEP 13560-970 São Carlos, SP. E-mail: reinaldo@cppse.embrapa.br
\end{abstract}

Resumo - O objetivo deste trabalho foi alterar o método centroide de avaliação da adaptabilidade e estabilidade fenotípica de genótipos, para deixá-lo com maior sentido biológico e melhorar aspectos quantitativos e qualitativos de sua análise. A alteração se deu pela adição de mais três ideótipos, definidos de acordo com valores médios dos genótipos nos ambientes. Foram utilizados dados provenientes de um experimento sobre produção de matéria seca de 92 genótipos de alfafa (Medicago sativa) realizado em blocos ao acaso, com duas repetições. Os genótipos foram submetidos a 20 cortes, no período de novembro de 2004 a junho de 2006. Cada corte foi considerado um ambiente. A inclusão dos ideótipos de maior sentido biológico (valores médios nos ambientes) resultou em uma dispersão gráfica em forma de uma seta voltada para a direita, na qual os genótipos mais produtivos ficaram próximos à ponta da seta. Com a alteração, apenas cinco genótipos foram classificados nas mesmas classes do método centroide original. A figura em forma de seta proporciona uma comparação direta dos genótipos, por meio da formação de um gradiente de produtividade. A alteração no método mantém a facilidade de interpretação dos resultados para a recomendação dos genótipos presente no método original e não permite duplicidade de interpretação dos resultados.

Termos para indexação: Medicago sativa, análise gráfica, componentes principais, interação genótipos x ambientes.

\section{Alteration of the centroid method to evaluate genotypic adaptability}

\begin{abstract}
The objective of this work was to modify the centroid method of evaluation of phenotypic adaptability and the phenotype stability of genotypes in order for the method to make greater biological sense and improve its quantitative and qualitative performance. The method was modified by means of the inclusion of three additional ideotypes defined in accordance with the genotypes' average yield in the environments tested. The alfalfa (Medicago sativa L.) forage yield of 92 genotypes was used. The trial had a randomized block design, with two replicates, and the data were used to test the method. The genotypes underwent 20 cuts, from November 2004 to June 2006. Each cut was considered an environment. The inclusion of ideotypes of greater biological average production in the environments produced an arrow-shaped graphical dispersion directed to the right in which the most productive genotypes were placed near the tip of the arrow. With the alteration only five genotypes were classified into the former classes of the original centroid method. The arrow-shaped figure allowed a direct comparison of genotypes throughout the productivity gradient. The alteration performed in the method preserved the easy interpretation of results for genotype recommendations of the original method, and does leaves no room for ambiguity in interpretation of the results.
\end{abstract}

Index terms: Medicago sativa, graphical analysis, principal components, genotype x environment interaction.

\section{Introdução}

Em programas de melhoramento, tanto na fase de seleção como na de recomendação de genótipos para plantio, a interação genótipo $\mathrm{x}$ ambiente constitui um grande problema para os melhoristas. Para contornar este problema, realizam-se análises de adaptabilidade e estabilidade, pelas quais se torna possível a identificação de genótipos de interesse para diversos estudos (Cruz et al., 2004). Como exemplo das inúmeras metodologias usadas para esse fim podem ser citados os métodos de Plaisted \& Peterson (1959) e Annicchiarico (1992), que se baseiam em análise da variância, e os de Finlay \& Wilkinson (1963) e Eberhart \& Russell (1966), que têm como princípio estatístico a análise de regressão linear simples. Métodos não paramétricos, como os desenvolvidos por Lin \& Binns (1988), Carneiro 
(1998) e Rocha et al. (2005) também são comumente empregados.

Silva \& Duarte (2006) utilizaram 11 métodos de avaliação da adaptabilidade e estabilidade da soja. Mohebodini et al. (2006) também utilizaram vários métodos para fazer estudo pormenorizado da interação genótipos $\mathrm{x}$ ambientes em 11 genótipos de lentilhas (Lens culinaris Medik). Mahammed \& Amri (2008) compararam 20 métodos paramétricos e não paramétricos na seleção de genótipos de Triticum durum.

O método centroide (Rocha et al., 2005) é um método não paramétrico que tem como objetivo facilitar a recomendação de genótipos, pois permite o direcionamento dos genótipos em relação à variação ambiental, dispensa a análise de vários parâmetros, como a que ocorre nos métodos baseados em regressão, e não possibilita a duplicidade de interpretação observada na metodologia de Lin \& Binns (1988). O método centroide consiste da comparação de valores de distância cartesiana entre os genótipos e quatro genótipos-referência, designados como ideótipos, estabelecidos com base nos dados experimentais com intuito de representarem os genótipos de máxima adaptabilidade geral e específica a ambientes favoráveis ou desfavoráveis e também os de mínima adaptabilidade.

Altoé (2007) utilizou o método centroide na análise de adaptabilidade e estabilidade em feijoeiro (Phaseolus vulgaris). Vasconcelos et al. (2008) fizeram uso do método centroide para auxiliar na avaliação e seleção de 92 genótipos de alfafa. Além desses, outros exemplos de uso do método centroide podem ser obtidos nos trabalhos de Barros et al. (2008), que avaliaram a adaptabilidade produtiva de 30 genótipos de soja em seis ambientes, e Pelúzio et al. (2008), que avaliaram o desempenho, a adaptabilidade e a estabilidade de genótipos de soja em quatro épocas de semeadura em Gurupi, TO.

Apesar da grande facilidade de recomendação proporcionada pelo método centroide, ele tem como pressuposto a existência de genótipos com desempenho elevado em determinado ambiente e muito baixo em outro. Esse fato não considera a possibilidade de existência de genótipos com desempenhos alto e médio ou baixo e médio. A polarização nas classes alto e baixo pode não ser observada em condições experimentais, principalmente em análises de caracteres quantitativos.
Assim, os ideótipos definidos geralmente não estão presentes no grupo de genótipos avaliados. A inclusão de maior número de ideótipos, definidos por meio dos valores médios dos genótipos nos diversos ambientes avaliados, pode fornecer ao método maior abrangência na caracterização genética, uma vez que, na natureza, o comportamento de qualquer variável quantitativa tende a seguir um valor médio de equilíbrio. A inclusão de ideótipos que apresentassem comportamento médio ao conjunto de genótipos avaliados conferiria maior sentido biológico ao método.

O objetivo deste trabalho foi alterar o método centroide de avaliação da adaptabilidade e estabilidade fenotípica de genótipos, para deixá-lo com maior sentido biológico e melhorar os aspectos quantitativos e qualitativos de sua análise.

\section{Material e Métodos}

O método centroide (Rocha et al., 2005) consiste da comparação de valores de distância cartesiana entre os genótipos e quatro referências preestabelecidas (ideótipos), criadas com base nos dados experimentais, cujos valores médios, em cada ambiente, são dados por: $\mathrm{C}_{1}$, ideótipo 1 (máxima adaptabilidade geral), cujos valores em cada ambiente são representados pelos máximos obtidos a partir do conjunto de genótipos estudado; $\mathrm{C}_{2}$, ideótipo 2 (máxima adaptabilidade específica a ambientes favoráveis), cujos valores nos ambientes favoráveis são representados pelos máximos e nos desfavoráveis, pelos mínimos obtidos no conjunto de genótipos estudado; $\mathrm{C}_{3}$, ideótipo 3 (máxima adaptabilidade específica a ambientes desfavoráveis), cujos valores nos ambientes favoráveis são representados pelos mínimos e nos desfavoráveis, pelos máximos obtidos no conjunto de genótipos estudado; $\mathrm{C}_{4}$, ideótipo 4 (mínima adaptabilidade), cujos valores, em cada ambiente, são representados pelos mínimos obtidos no conjunto de genótipos estudado.

Para utilização do método centroide, os ambientes devem ser classificados em favoráveis e desfavoráveis utilizando o índice ambiental proposto por Finlay \& Wilkinson (1963): $I_{j}=\frac{1}{g} \sum_{i} Y_{i j}-\frac{1}{a g} Y_{\text {.., em que: } Y_{i j}}$ é a média do genótipo i no ambiente $\mathrm{j}$; $\mathrm{Y}_{. .}$é o total das observações; a é o número de ambientes; e g é o número de genótipos. 
Após a classificação dos ambientes e criação dos pontos referenciais representativos dos ideótipos (centroides), utiliza-se a análise de componentes principais considerando, na matriz de médias de dimensão $\mathrm{g} \times \mathrm{a}$, quatro linhas adicionais correspondentes aos ideótipos estabelecidos. A partir desses genótipos $(\mathrm{g}+4)$ é feita a análise de componentes principais de maneira usual, obtendo-se escores utilizados na representação gráfica. A posição dos genótipos em relação aos centroides (ideótipos) no gráfico de dispersão e os valores de distância cartesiana entre os pontos (genótipos) e cada um dos quatro centroides possibilitam a sua classificação quanto à adaptabilidade e à estabilidade. Uma medida de probabilidade espacial é calculada com uso do inverso da distância entre um tratamento e os quatro ideótipos:

$P_{d(i, k)}=\frac{\left(\frac{1}{d_{i k}}\right)}{\sum_{i} \frac{1}{d_{i k}}}$, em que: $P_{d(i, k)}$ é a probabilidade de apresentar padrão de estabilidade semelhante ao k-ésimo centroide e $\mathrm{d}_{\mathrm{ik}}$ é a distância do i-ésimo genótipo ao k-ésimo centroide no plano gerado a partir da análise de componentes principais.

A modificação proposta neste trabalho consistiu da adição de três novos ideótipos (centroides), também criados com base nos dados experimentais, cujos valores médios para cada ambiente seriam dados por: $\mathrm{C}_{5}$, ideótipo 5 (média adaptabilidade geral), cujos valores, em cada ambiente, são representados pelas médias obtidas pelo conjunto de genótipos estudado; $\mathrm{C}_{6}$, ideótipo 6 (média adaptabilidade específica a ambientes favoráveis), cujos valores nos ambientes favoráveis são representados pelos valores máximos e, nos desfavoráveis, pelas médias obtidas pelo conjunto de genótipos estudado; $\mathrm{C}_{7}$, ideótipo 7 (média adaptabilidade específica a ambientes desfavoráveis), cujos valores, nos ambientes favoráveis, são representados pelas médias e, nos desfavoráveis, pelos valores máximos obtidos pelo conjunto de genótipos estudado. A partir desse ponto, procede-se à análise como no método centroide original.

Assim como no método centroide original (Rocha et al., 2005), o conceito de adaptabilidade e estabilidade na metodologia aqui proposta difere das demais metodologias existentes na literatura. Os genótipos de máxima e média adaptação específica não são aqueles que apresentam bom desempenho nos grupos de ambientes favoráveis ou desfavoráveis, mas sim os genótipos que apresentam valores máximos ou médios para determinado grupo de ambientes (favoráveis e desfavoráveis) e mínimo para o outro conjunto. Após a classificação dos ambientes e criação dos sete pontos ou dos quatro pontos referenciais representativos dos ideótipos (centroides), procede-se à análise de componentes principais para obtenção dos escores utilizados na representação gráfica.

Foram utilizados dados provenientes de um experimento de avaliação da produção de matéria seca de 92 genótipos de alfafa em 20 cortes. O delineamento utilizado nesse experimento foi o de blocos ao acaso, com duas repetições. $\mathrm{O}$ experimento foi conduzido pela Embrapa Pecuária Sudeste, para desenvolvimento de genótipos de alfafa adaptados aos diferentes ecossistemas brasileiros. No presente trabalho, consideraram-se os cortes como representativos de diferentes condições ambientais, já que foram realizados em diferentes épocas durante o período de novembro de 2004 a junho de 2006. A avaliação da adaptabilidade e estabilidade dos genótipos foi também realizada pelas metodologias do método centroide original e pela metodologia proposta por Lin \& Binns (1988).

As análises estatísticas foram realizadas com auxílio do aplicativo computacional GENES (Cruz, 2006).

\section{Resultados e Discussão}

A adição dos três novos centroides fez com que sua configuração conjunta tivesse o formato de uma seta (Figura 1).

A análise da variância para o caráter produção de matéria seca (Tabela 1) revelou a existência de diferenças significativas entre as médias dos genótipos de alfafa e a existência da interação genótipos x cortes. A significância da interação entre cultivares $\mathrm{x}$ cortes indica que as cultivares apresentaram desempenho diferenciado frente às diferentes condições ambientais. Assim fez-se necessário um estudo pormenorizado do comportamento das cultivares frente a essas variações, por meio da análise de adaptabilidade e estabilidade.

O percentual de variância acumulada pelos dois primeiros componentes principais foi de 76,38\% (Tabela 2), valor que, após uma revisão sobre utilização de componentes principais nas diversas áreas de aplicação, é tido como suficiente para interpretação dos dados com sucesso (Johnson \& Wichern, 1999; Melém Júnior et al., 2008). 
A Figura 2 apresenta a dispersão gráfica dos dois primeiros componentes principais para os 92 genótipos nos 20 cortes (ambientes). Percebe-se a formação de um gradiente em forma de seta para a direita, que possibilita a inferência quantitativa da adaptabilidade por meio de uma inspeção visual adequada, de modo que os genótipos mais próximos à ponta da seta são mais produtivos e os mais próximos à base da seta, menos produtivos. Além

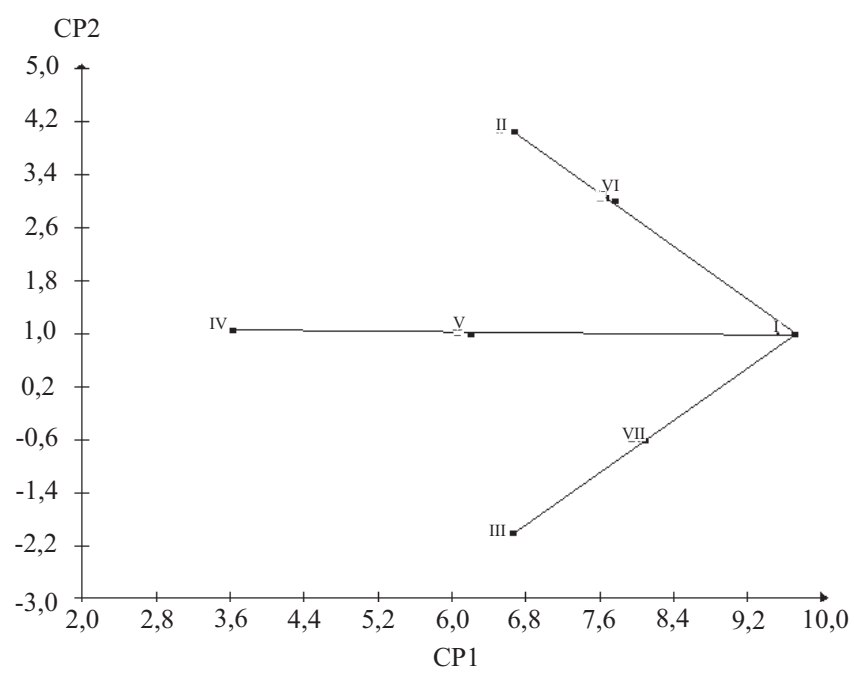

Figura 1. Configuração obtida a partir dos sete centroides. Os sete pontos numerados com algarismos romanos representam os ideótipos. I, máxima adaptabilidade geral; II, máxima adaptabilidade específica a ambientes favoráveis; III, máxima adaptabilidade específica a ambientes desfavoráveis; IV, mínima adaptabilidade; V, média adaptabilidade geral; VI, média adaptabilidade específica a ambientes favoráveis; VII, média adaptabilidade específica a ambientes desfavoráveis. CP1: componente principal 1; CP2: componente principal 2.

Tabela 1. Análise da variância para o caráter produção de matéria seca em 92 cultivares de alfafa no período de novembro de 2004 a junho de 2006.

\begin{tabular}{lrc}
\hline Fontes de variação & \multicolumn{1}{c}{ GL } & Quadrados médios \\
\hline Blocos & 1 & $2.002 .415,43$ \\
Cultivar (Cv) & 91 & $1.384 .475,75^{*}$ \\
Erro a & 91 & $574.269,72$ \\
Corte (Co) & 19 & $62.331 .022,56^{*}$ \\
Erro b & 19 & $946.917,67$ \\
Interação (Cv x Co) & 1.729 & $60.682,46^{* *}$ \\
Erro c & 1.729 & $55.851,26$ \\
CVa (\%) & 64,39 & \\
CVb (\%) & 82,69 & \\
CVc (\%) & 20,08 & \\
Média & 1.176 & \\
\hline
\end{tabular}

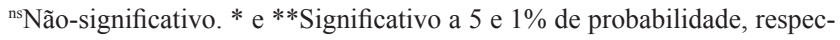
tivamente, pelo teste $\mathrm{F}$. disso, na medida em que os genótipos se afastam do segmento de reta, para cima ou para baixo, eles podem ser classificados como adaptáveis a ambientes favoráveis e desfavoráveis, respectivamente.

Tabela 2. Estimativas dos autovalores e fração cumulativa da variância explicada pelos componentes principais.

\begin{tabular}{ccc}
\hline Raiz & Raiz $(\%)$ & \% Acumulada \\
\hline 11,52 & 57,63 & 57,63 \\
3,75 & 18,75 & 76,38 \\
1,03 & 5,16 & 81,54 \\
0,69 & 3,42 & 84,96 \\
0,45 & 2,26 & 87,23 \\
0,40 & 1,98 & 89,21 \\
0,32 & 1,62 & 90,83 \\
0,28 & 1,42 & 92,25 \\
0,27 & 1,36 & 93,61 \\
0,25 & 1,24 & 94,85 \\
0,18 & 0,88 & 95,72 \\
0,15 & 0,76 & 96,48 \\
0,13 & 0,64 & 97,13 \\
0,13 & 0,62 & 97,75 \\
0,11 & 0,54 & 98,29 \\
0,10 & 0,49 & 98,78 \\
0,09 & 0,47 & 99,25 \\
0,07 & 0,34 & 99,59 \\
0,05 & 0,24 & 99,83 \\
0,03 & 0,17 & 100,00 \\
\hline
\end{tabular}

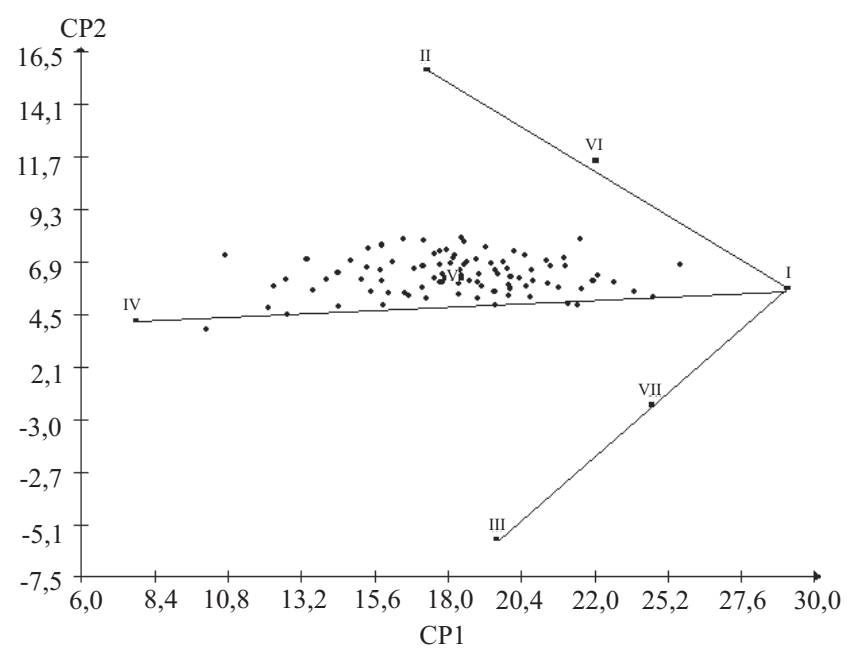

Figura 2. Dispersão gráfica dos dois primeiros componentes principais dos 92 genótipos de alfafa, para variável produção de matéria seca em 20 ambientes (cortes realizados no período de novembro de 2004 a junho de 2006). Os sete pontos numerados com algarismos romanos representam os ideótipos. I, máxima adaptabilidade geral; II, máxima adaptabilidade específica a ambientes favoráveis; III, máxima adaptabilidade específica a ambientes desfavoráveis; IV, mínima adaptabilidade; V, média adaptabilidade geral; VI, média adaptabilidade específica a ambientes favoráveis; VII, média adaptabilidade específica a ambientes desfavoráveis. CP1, componente principal 1; CP2, componente principal 2. 
A Tabela 3 mostra a classificação dos genótipos em relação ao método centroide original. Dos 92 genótipos, 27 foram classificados como de mínima adaptabilidade geral (classe IV), sendo passíveis de descarte. Os cinco genótipos com maior probabilidade de pertencer a essa classe foram Candombe $(0,65)$, Siriver $(0,52)$, Coronado (0,51), Tango $(0,50)$ e WL $414(0,47)$. Sessenta e dois genótipos foram classificados como de máxima adaptabilidade a ambientes favoráveis e aqueles com maior probabilidade de pertencer a essa classe foram Kern $(0,37)$, Activa (0,37), Prointa Lujan $(0,37)$, Hunterfield $(0,36)$ e Sundor $(0,36)$. Os genótipos classificados como de adaptabilidade máxima geral foram $5939(0,30)$, P $105(0,31)$ e WL $516(0,31)$.

A análise dos dados pelo método centroide com os pontos adicionais (Tabela 3) indicou que, dos 92 genótipos, 84 foram classificados como de média adaptabilidade geral (classe V). Entre esses, os cinco genótipos com maior probabilidade de pertencer à classe V foram Siriver $2(0,58)$, Perla SP INTA $(0,58)$, Alfa $200(0,56)$, SPS $6550(0,54)$ e WL $442(0,54)$.

Tabela 3. Estimativa dos parâmetros de adaptabilidade e estabilidade obtidos pelos métodos centroide original (MC), centroide com pontos adicionais (MCPA) e Lin \& Binns, para o caráter produção de matéria seca de alfafa ${ }^{(1)}$.

\begin{tabular}{|c|c|c|c|c|c|c|c|c|c|c|c|}
\hline \multirow[t]{2}{*}{ Cultivares } & \multirow[t]{2}{*}{ Média } & \multicolumn{2}{|c|}{$\mathrm{MC}$} & \multicolumn{2}{|c|}{ MCPA } & \multicolumn{6}{|c|}{ Lin e Binns (1988) } \\
\hline & & Class. & Prob. & Class. & Prob. & Class. & $\mathrm{P}_{\mathrm{i}}$ & Class. & $\mathrm{P}_{\mathrm{i}}$ fav. & Class. & $\mathrm{P}_{\mathrm{i}}$ desf. \\
\hline 1-Winter & $1.002,85$ & IV & 0,34 & $\mathrm{~V}$ & 0,36 & 45 & $235.472,34$ & 41 & $37.180,94$ & 45 & $223.575,45$ \\
\hline 2-SPS 6550 & $1.133,70$ & II & 0,33 & $\mathrm{~V}$ & 0,54 & 41 & $262.956,04$ & 37 & $60.064,99$ & 66 & $415.696,31$ \\
\hline 3-Primavera & $1.052,28$ & IV & 0,35 & $\mathrm{~V}$ & 0,36 & 17 & $292.152,13$ & 17 & $78.710,83$ & 41 & $447.681,11$ \\
\hline 4-LE N 1 & $1.184,56$ & II & 0,34 & $\mathrm{~V}$ & 0,50 & 32 & $306.196,73$ & 32 & $84.947,67$ & 17 & $466.785,92$ \\
\hline 5-Trindade 87 & $1.467,20$ & II & 0,30 & $\mathrm{~V}$ & 0,28 & 63 & $329.223,96$ & 63 & $95.972,39$ & 32 & $487.218,69$ \\
\hline 6-LE N 2 & $1.352,26$ & II & 0,33 & $\mathrm{~V}$ & 0,35 & 5 & $337.543,51$ & 76 & $104.554,55$ & 18 & $503.535,04$ \\
\hline 7-LE Semit 711 & $1.140,25$ & II & 0,35 & $\mathrm{~V}$ & 0,40 & 33 & $339.018,09$ & 33 & $107.414,39$ & 9 & $510.865,03$ \\
\hline 8-Topper & $1.144,19$ & IV & 0,31 & $\mathrm{~V}$ & 0,33 & 66 & $340.669,40$ & 38 & $111.939,38$ & 63 & $520.066,15$ \\
\hline 9-LE N 3 & $1.394,53$ & II & 0,29 & $\mathrm{~V}$ & 0,30 & 76 & $350.884,22$ & 5 & $112.580,47$ & 5 & $521.604,17$ \\
\hline 10-sequel HR & $1.306,67$ & II & 0,35 & $\mathrm{~V}$ & 0,41 & 31 & $356.239,01$ & 10 & $126.967,34$ & 33 & $528.512,02$ \\
\hline $11-L E$ N 4 & $1.382,65$ & II & 0,31 & $\mathrm{~V}$ & 0,31 & 9 & $357.513,50$ & 36 & $129.803,04$ & 31 & $541.067,87$ \\
\hline 12-Victoria INTA & $1.213,57$ & II & 0,31 & $\mathrm{~V}$ & 0,49 & 18 & $358.002,24$ & 65 & $129.983,92$ & 76 & $552.426,67$ \\
\hline 13-Monarca INTA & $1.189,03$ & II & 0,33 & $\mathrm{~V}$ & 0,54 & 38 & $359.788,25$ & 31 & $130.337,06$ & 38 & $562.573,68$ \\
\hline 14-Bárbara INTA & $1.315,94$ & II & 0,30 & V & 0,40 & 37 & $369.277,02$ & 11 & $133.563,51$ & 11 & $577.633,17$ \\
\hline 15-Primavera 1 & $1.196,40$ & II & 0,32 & $\mathrm{~V}$ & 0,46 & 11 & $377.801,82$ & 6 & $135.995,01$ & 6 & $580.475,23$ \\
\hline 16-Aca 900 & $1.273,35$ & II & 0,30 & $\mathrm{~V}$ & 0,44 & 6 & $380.459,13$ & 43 & $138.401,46$ & 36 & $590.067,59$ \\
\hline 17-5 939 & $1.574,60$ & $\mathrm{I}$ & 0,30 & $\mathrm{~V}$ & 0,22 & 36 & $382.948,54$ & 30 & $145.691,65$ & 61 & $590.308,28$ \\
\hline 18-WL 612 & $1.399,08$ & II & 0,28 & $\mathrm{~V}$ & 0,32 & 59 & $395.931,76$ & 59 & $155.565,54$ & 59 & $592.595,03$ \\
\hline 19-Medina & $1.251,65$ & II & 0,29 & V & 0,42 & 65 & $398.478,66$ & 58 & $160.601,77$ & 14 & $593.962,81$ \\
\hline 20-N 910 & $1.183,79$ & II & 0,31 & V & 0,44 & 61 & $399.834,18$ & 61 & $167.032,49$ & 78 & $598.936,43$ \\
\hline 21-Coronado & 793,91 & IV & 0,51 & IV & 0,28 & 14 & $414.808,50$ & 9 & $170.083,85$ & 19 & $601.920,19$ \\
\hline 22-Eterna & 835,14 & IV & 0,46 & $\mathrm{~V}$ & 0,26 & 10 & $425.684,84$ & 75 & $174.267,94$ & 16 & $610.069,93$ \\
\hline 23-DK 193 & 915,61 & IV & 0,39 & V & 0,28 & 43 & $426.010,42$ & 40 & $176.812,57$ & 65 & $618.156,18$ \\
\hline 24-Candombe & 651,02 & IV & 0,65 & IV & 0,45 & 78 & $431.208,54$ & 77 & $178.856,38$ & 60 & $618.907,58$ \\
\hline 25-WL 414 & 830,55 & IV & 0,47 & IV & 0,26 & 58 & $434.807,11$ & 34 & $179.851,14$ & 37 & $622.268,69$ \\
\hline 26-Crioula & $1.033,33$ & IV & 0,33 & $\mathrm{~V}$ & 0,42 & 60 & $437.716,42$ & 18 & $180.128,82$ & 49 & $625.826,26$ \\
\hline 27-LE Semit 7111 & 991,08 & IV & 0,37 & $\mathrm{~V}$ & 0,37 & 16 & $437.758,62$ & 42 & $186.655,01$ & 29 & $629.687,61$ \\
\hline 28-DK 181 & $1.053,43$ & II & 0,33 & $\mathrm{~V}$ & 0,42 & 34 & $437.994,65$ & 83 & $188.201,72$ & 52 & $633.972,21$ \\
\hline $29-5929$ & $1.264,67$ & II & 0,31 & $\mathrm{~V}$ & 0,46 & 80 & $439.337,39$ & 35 & $189.398,13$ & 80 & $642.935,34$ \\
\hline 30-Activa & $1.257,95$ & II & 0,37 & $\mathrm{~V}$ & 0,33 & 77 & $439.439,26$ & 80 & $190.495,44$ & 39 & $642.976,43$ \\
\hline 31-sequel 2 & $1.411,09$ & II & 0,30 & $\mathrm{~V}$ & 0,31 & 35 & $443.438,82$ & 14 & $195.842,11$ & 34 & $649.202,98$ \\
\hline 32-Califónia 60 & $1.525,05$ & II & 0,29 & $\mathrm{~V}$ & 0,24 & 39 & $443.590,45$ & 39 & $199.896,47$ & 35 & $651.290,30$ \\
\hline 33-Cuf 101 & $1.437,20$ & II & 0,31 & $\mathrm{~V}$ & 0,30 & 75 & $444.914,75$ & 57 & $200.187,51$ & 77 & $652.643,42$ \\
\hline $34-58$ N 58 & $1.263,82$ & II & 0,33 & $\mathrm{~V}$ & 0,46 & 19 & $444.991,67$ & 64 & $202.143,70$ & 58 & $659.156,93$ \\
\hline 35-Diamind & $1.277,02$ & II & 0,31 & $\mathrm{~V}$ & 0,42 & 52 & $446.558,49$ & 13 & $210.902,64$ & 43 & $661.326,84$ \\
\hline 36-Aurora & $1.336,74$ & II & 0,33 & $\mathrm{~V}$ & 0,35 & 29 & $448.317,80$ & 4 & $211.621,99$ & 12 & $662.027,33$ \\
\hline 37-Sundor & $1.428,93$ & II & 0,36 & VI & 0,29 & 49 & $449.907,69$ & 55 & $216.249,71$ & 75 & $666.353,04$ \\
\hline 38-Springfield & $1.383,94$ & II & 0,33 & $\mathrm{~V}$ & 0,30 & 30 & $464.164,23$ & 60 & $216.260,57$ & 10 & $670.090,07$ \\
\hline 39-Sutter & $1.211,48$ & II & 0,33 & $\mathrm{~V}$ & 0,44 & 12 & $477.254,08$ & 52 & $217.497,27$ & 79 & $672.229,49$ \\
\hline 40-Hunterfield & $1.189,43$ & II & 0,36 & $\mathrm{~V}$ & 0,37 & 62 & $479.016,65$ & 7 & $222.260,92$ & 62 & $672.375,93$ \\
\hline 41-P 105 & $1.622,76$ & $\mathrm{I}$ & 0,31 & VI & 0,25 & 79 & $481.318,79$ & 44 & $222.944,05$ & 50 & $682.165,17$ \\
\hline 42-Prointa Patricia & $1.184,07$ & II & 0,35 & $\mathrm{~V}$ & 0,47 & 50 & $482.536,00$ & 78 & $226.207,79$ & 20 & $687.557,17$ \\
\hline 43-Flórida 77 & $1.288,05$ & II & 0,35 & V & 0,38 & 15 & $491.489,51$ & 29 & $226.643,59$ & 8 & $690.074,52$ \\
\hline 44-Siriver 2 & $1.185,93$ & II & 0,33 & $\mathrm{~V}$ & 0,58 & 42 & $491.885,34$ & 16 & $227.155,91$ & 15 & $705.311,63$ \\
\hline 45-WL 516 & $1.334,34$ & $\mathrm{I}$ & 0,31 & VII & 0,22 & 40 & $492.310,72$ & 15 & $230.151,35$ & 74 & $717.152,06$ \\
\hline
\end{tabular}

Continua... 
Os genótipos Sundor $(0,29)$ e P $105(0,25)$ foram classificados como de adaptabilidade média a ambientes favoráveis e o genótipo WL $516(0,22)$ foi classificado como de adaptabilidade média a ambientes desfavoráveis. Já os genótipos Candombe $(0,45)$, Siriver $(0,31)$, Coronado $(0,28)$, Tango $(0,28)$ e WL $414(0,26)$ foram classificados como de mínima adaptabilidade e são passíveis de descarte.

Percebe-se que a adição dos centroides fez com que apenas cinco genótipos fossem classificados na classe de mínima adaptabilidade (IV) do método original, isto é, apresentaram comportamento extremo. Esse resultado é indicativo de que os centroides adicionados conferiram ao método maior sentido biológico. Além disso, a adição dos centroides contribuiu para a melhoria do método em termos quantitativos e qualitativos da análise, uma vez que, dos 27 genótipos classificados como de mínima adaptabilidade geral no método centroide original, apenas cinco foram classificados da mesma forma pelo método aqui proposto. Isto é, houve uma perda de 22 genótipos que poderiam ser de interesse para o estudo de adaptabilidade fenotípica quando foi feita a análise pelo método original.

Tabela 3. Continuação.

\begin{tabular}{|c|c|c|c|c|c|c|c|c|c|c|c|}
\hline \multirow[t]{2}{*}{ Cultivares } & \multirow[t]{2}{*}{ Média } & \multicolumn{2}{|c|}{$\mathrm{MC}$} & \multicolumn{2}{|c|}{ MCPA } & \multicolumn{6}{|c|}{ Lin e Binns (1988) } \\
\hline & & Class. & Prob. & Class. & Prob. & Class. & $\mathrm{P}_{\mathrm{i}}$ & Class. & $\mathrm{P}_{\mathrm{i}}$ fav. & Class. & $\mathrm{P}_{\mathrm{i}}$ desf. \\
\hline 46-Tahoe & $1.041,09$ & II & 0,34 & $\mathrm{~V}$ & 0,41 & 44 & $498.105,18$ & 86 & $230.334,96$ & 81 & $719.196,74$ \\
\hline 47-Esmeralda & $1.081,97$ & IV & 0,32 & V & 0,47 & 83 & $502.460,53$ & 82 & $231.524,21$ & 71 & $720.367,64$ \\
\hline 48-DK 167 & $1.122,71$ & II & 0,34 & V & 0,50 & 4 & $503.848,43$ & 49 & $234.896,10$ & 44 & $723.237,02$ \\
\hline 49-DK 177 & $1.274,64$ & II & 0,31 & $\mathrm{~V}$ & 0,38 & 20 & $505.046,01$ & 50 & $238.544,80$ & 30 & $724.732,69$ \\
\hline $50-5683$ & $1.205,99$ & II & 0,32 & $\mathrm{~V}$ & 0,51 & 13 & $508.807,97$ & 62 & $242.688,65$ & 56 & $725.279,11$ \\
\hline 51-WL 4141 & $1.010,34$ & IV & 0,34 & V & 0,38 & 82 & $513.204,27$ & 79 & $247.983,49$ & 90 & $727.153,04$ \\
\hline 52-Express & $1.267,47$ & II & 0,31 & V & 0,41 & 57 & $515.157,44$ & 66 & $248.969,83$ & 42 & $741.619,25$ \\
\hline 53-F 708 & $1.108,73$ & II & 0,32 & $\mathrm{~V}$ & 0,51 & 56 & $519.602,71$ & 45 & $250.012,98$ & 54 & $741.980,19$ \\
\hline 54-Perla INTA & $1.129,40$ & II & 0,31 & $\mathrm{~V}$ & 0,58 & 90 & $520.874,07$ & 48 & $250.084,75$ & 4 & $742.942,78$ \\
\hline 55-Prointa Lujan & $1.088,19$ & II & 0,37 & $\mathrm{~V}$ & 0,39 & 81 & $531.261,57$ & 12 & $251.420,10$ & 82 & $743.669,78$ \\
\hline 56-DK 166 & $1.150,30$ & II & 0,32 & $\mathrm{~V}$ & 0,48 & 54 & $535.170,87$ & 19 & $253.190,14$ & 40 & $750.445,57$ \\
\hline 57-Platino & $1.179,55$ & II & 0,35 & V & 0,46 & 2 & $547.132,91$ & 2 & $263.962,50$ & 13 & $752.548,70$ \\
\hline 58-Maxidor & $1.274,52$ & II & 0,34 & $\mathrm{~V}$ & 0,43 & 71 & $549.586,81$ & 56 & $268.220,46$ & 87 & $752.835,68$ \\
\hline 59-Amerigraze701 & $1.355,01$ & II & 0,31 & $\mathrm{~V}$ & 0,36 & 86 & $554.039,38$ & 90 & $268.755,32$ & 88 & $757.295,23$ \\
\hline 60-13 R Supreme & $1.270,49$ & II & 0,30 & V & 0,46 & 8 & $554.619,87$ & 46 & $272.116,87$ & 83 & $759.581,37$ \\
\hline 61-Pecos & $1.322,88$ & II & 0,31 & $\mathrm{~V}$ & 0,41 & 53 & $560.568,51$ & 53 & $274.909,98$ & 57 & $772.860,10$ \\
\hline 62-Califórnia 50 & $1.211,35$ & II & 0,31 & $\mathrm{~V}$ & 0,50 & 48 & $566.289,10$ & 20 & $281.976,82$ & 84 & $777.777,82$ \\
\hline 63-Maricopa & $1.459,60$ & II & 0,31 & $\mathrm{~V}$ & 0,27 & 64 & $571.848,02$ & 54 & $282.403,92$ & 2 & $778.817,79$ \\
\hline 64-Kern & $1.120,37$ & II & 0,37 & $\mathrm{~V}$ & 0,36 & 7 & $581.177,86$ & 28 & $295.297,47$ & 73 & $783.982,29$ \\
\hline 65-Costera INTA & $1.357,18$ & II & 0,33 & $\mathrm{~V}$ & 0,33 & 84 & $582.795,17$ & 81 & $301.563,02$ & 3 & $791.458,63$ \\
\hline $66-F 686$ & $1.271,35$ & II & 0,27 & $\mathrm{~V}$ & 0,32 & 55 & $584.800,90$ & 1 & $307.733,23$ & 53 & $794.289,13$ \\
\hline 67-Monarca & $1.034,20$ & IV & 0,34 & V & 0,43 & 74 & $587.982,62$ & 47 & $319.583,61$ & 67 & $811.667,00$ \\
\hline 68-Patrícia & 971,81 & IV & 0,36 & $\mathrm{~V}$ & 0,35 & 47 & $598.152,14$ & 26 & $328.898,10$ & 86 & $818.888,46$ \\
\hline 69-Tango & 804,51 & IV & 0,50 & IV & 0,28 & 88 & $601.359,98$ & 51 & $333.057,38$ & 48 & $825.001,75$ \\
\hline 70-Bárbara & 872,00 & IV & 0,44 & $\mathrm{~V}$ & 0,26 & 73 & $605.967,74$ & 71 & $340.854,69$ & 47 & $826.071,85$ \\
\hline 71-Rio Grande & $1.116,73$ & IV & 0,31 & $\mathrm{~V}$ & 0,45 & 67 & $607.833,35$ & 84 & $344.483,05$ & 27 & $848.775,18$ \\
\hline 72-Key II & 904,77 & IV & 0,37 & $\mathrm{~V}$ & 0,28 & 28 & $611.185,60$ & 67 & $358.703,35$ & 89 & $854.982,88$ \\
\hline 73-Gala & $1.041,23$ & IV & 0,34 & $\mathrm{~V}$ & 0,43 & 46 & $621.245,02$ & 68 & $378.522,17$ & 85 & $867.773,36$ \\
\hline 74-Lujan & $1.131,27$ & IV & 0,31 & $\mathrm{~V}$ & 0,30 & 26 & $627.654,51$ & 73 & $388.394,41$ & 28 & $869.639,53$ \\
\hline 75-Perla & $1.252,13$ & II & 0,34 & $\mathrm{~V}$ & 0,46 & 51 & $635.286,40$ & 8 & $389.064,19$ & 26 & $872.091,56$ \\
\hline $76-5683 \mathrm{~L}$ & $1.404,40$ & II & 0,32 & V & 0,30 & 87 & $636.339,60$ & 72 & $405.143,81$ & 64 & $874.333,38$ \\
\hline 77-Victoria & $1.283,16$ & II & 0,32 & V & 0,38 & 3 & $636.876,92$ & 88 & $410.772,45$ & 7 & $874.837,17$ \\
\hline 78-DK 194 & $1.300,29$ & II & 0,30 & V & 0,37 & 1 & $657.604,37$ & 74 & $430.108,85$ & 51 & $882.564,69$ \\
\hline 79-WL 442 & $1.194,41$ & II & 0,31 & V & 0,54 & 27 & $669.589,00$ & 3 & $447.943,73$ & 55 & $886.342,78$ \\
\hline 80-P 30 & $1.259,66$ & II & 0,32 & V & 0,48 & 85 & $681.077,22$ & 91 & $447.965,36$ & 46 & $906.895,33$ \\
\hline 81-P 5715 & $1.141,71$ & II & 0,30 & V & 0,54 & 68 & $686.745,10$ & 27 & $450.583,67$ & 91 & $915.265,24$ \\
\hline 82-Alfa 200 & $1.165,58$ & II & 0,33 & V & 0,56 & 91 & $704.980,30$ & 85 & $452.893,05$ & 68 & $938.927,49$ \\
\hline 83-Aca 901 & $1.200,67$ & II & 0,35 & V & 0,36 & 89 & $715.246,96$ & 87 & $493.955,50$ & 1 & $943.862,57$ \\
\hline 84-Gapp 969 & $1.093,06$ & IV & 0,33 & V & 0,49 & 72 & $761.389,50$ & 23 & $529.952,30$ & 25 & $957.100,39$ \\
\hline 85-Rocio & 974,53 & IV & 0,39 & V & 0,35 & 23 & $776.073,87$ & 89 & $544.458,62$ & 70 & $970.024,80$ \\
\hline 86-GT 13 R Plus & $1.143,14$ & II & 0,35 & V & 0,46 & 70 & $792.140,75$ & 22 & $566.244,10$ & 23 & $977.446,06$ \\
\hline 87-WL 525 & $1.013,74$ & IV & 0,37 & V & 0,36 & 25 & $818.314,66$ & 70 & $574.726,91$ & 21 & $1.028 .229,01$ \\
\hline 88-Sequel & $1.056,44$ & IV & 0,35 & V & 0,42 & 22 & $823.283,57$ & 92 & $619.670,22$ & 22 & $1.033 .588,58$ \\
\hline 89-DK $187 \mathrm{R}$ & 934,30 & IV & 0,39 & V & 0,29 & 69 & $857.406,25$ & 69 & $630.696,17$ & 69 & $1.042 .896,33$ \\
\hline 90-Pinto & $1.148,74$ & II & 0,32 & V & 0,51 & 21 & $868.788,76$ & 25 & $648.687,66$ & 72 & $1.0528 .63,23$ \\
\hline 91-Bacana & 939,33 & IV & 0,39 & $\mathrm{~V}$ & 0,33 & 92 & $969.241,67$ & 21 & $673.917,34$ & 24 & $1.109 .434,51$ \\
\hline 92-Siriver & 718,90 & IV & 0,52 & IV & 0,31 & 24 & $1059.743,34$ & 24 & $999.009,68$ & 92 & $1.255 .254,68$ \\
\hline
\end{tabular}

${ }^{(1)}$ Class., classificação; Prob., probabilidade; $\mathrm{P}_{\mathrm{i}}$, estimativa da estabilidade e adaptabilidade do cultivar i para todos ambientes; $\mathrm{P}_{\mathrm{i}}$ fav., estimativa da estabilidade e adaptabilidade do cultivar i para ambientes favoráveis; $\mathrm{P}_{\mathrm{i}}$ desf., estimativa da estabilidade e adaptabilidade do cultivar i para ambientes desfavoráveis. 
Esse resultado é justificado pelo fato de que, no método original, a comparação é feita apenas com ideótipos de comportamentos polarizados (extremos).

Com base nos resultados obtidos pela metodologia de Lin \& Binns (1988) (Tabela 2), os genótipos WL 516, P 105, 5939, Califórnia 60 e Maricopa foram classificados como de adaptabilidade geral (menor $\mathrm{P}_{\mathrm{i}}$ ). Foram classificados como adaptáveis a ambientes favoráveis os genótipos P 105, Sundor, 5 939, Califórnia 60 e Maricopa e, como adaptáveis a ambientes desfavoráveis, WL 516, F686, P 105, 5939 e Califórnia 60. Todos esses genótipos apresentam valores de média superiores à média geral, o que indica um comportamento superior à maioria dos genótipos avaliados.

Avaliando os resultados de ambos os métodos, percebe-se maior facilidade de análise e interpretação da adaptabilidade pelo método centroide com pontos adicionais em relação à metodologia de Lin \& Binns (1988). Esta facilidade de interpretação se dá pela não ocorrência de possíveis duplicidades de indicações, como a ocorrida com o uso do método de Lin \& Binns (1988) para o genótipo WL 516, que foi classificado como de adaptabilidade geral e adaptável a ambientes desfavoráveis.

\section{Conclusões}

1. A modificação proposta no método centroide de avaliação da adaptabilidade e estabilidade fenotípica de genótipos dá maior sentido biológico ao método, o que contribui para a melhoria da análise nos aspectos quantitativo e qualitativo.

2. O gráfico fornecido pelo método possibilita comparação direta em relação a um gradiente de produtividade, onde genótipos mais próximos à ponta da seta são mais produtivos.

3. A alteração no método mantém a facilidade de interpretação dos resultados para a recomendação dos genótipos presente no método original e não possibilita duplicidade de interpretação.

\section{Referências}

ALTOÉ, M. Comportamento de genótipos de feijoeiro sob cultivo orgânico. 2007. 63p. Dissertação (Mestrado) - Universidade Federal do Espírito Santo, Vitória.

ANNICCHIARICO, P. Cultivar adaptation and recommendation from alfalfa trials in Northern Italy. Journal of Genetics and Plant Breeding, v.46, p.269-278, 1992.
BARROS, H.B.; SEDIYAMA, T.; TEIXEIRA, R. de C.; CRUZ, C.D. Análises paramétricas e não-paramétricas para determinação da adaptabilidade e estabilidade de genótipos de soja. Scientia Agraria, v.9, p.299-309, 2008.

CARNEIRO, P.C.S. Novas metodologias de análise da adaptabilidade e estabilidade de comportamento. 1998. 168p. Tese (Doutorado) - Universidade Federal de Viçosa, Viçosa.

CRUZ, C.D. Programa Genes: biometria. Viçosa: UFV, 2006. 382p.

CRUZ, C.D.; REGAZZI, A.J.; CARNEIRO, P.C.S. Modelos biométricos aplicados ao melhoramento genético. 3.ed. Viçosa: UFV, 2004. v.1. 480p.

EBERHART, S.A.; RUSSELL, W.A. Stability parameters for comparing varieties. Crop Science, v.6, p.36-40, 1966.

FINLAY, K.W.; WILKINSON, G.N. The analysis of adaptation in a plant-breeding programme. Australian Journal of Agricultural Research, v.14, p.742-754, 1963.

JOHNSON, R.A.; WICHERN, D.W. Applied multivariate statistical analysis. $4^{\text {th }}$ ed. New Jersey: Prentice-Hall, 1999. 815p.

LIN, C.S.; BINNS, M.R. A superiority measure of cultivar performance for cultivar $\mathrm{x}$ location data. Canadian Journal of Plant Science, v.68, p.193-198, 1988.

MAHAMMADI, R.; AMRI, A. Comparison of parametric and non-parametric methods for selecting stable and adapted durum wheat genotypes in variable environments. Euphytica, v.159, p.419-432, 2008.

MELÉM JÚNIOR, N.J.; FONSECA, I.C. de B.; BRITO, O.R.; DECAËNS, T.; CARNEIRO, M.M.; MATOS, M. de F.A. de; GUEDES, M.C.; QUEIROZ, J.A.L. de; BARROSO, K. de O. Análise de componentes principais para avaliação de resultados analíticos da fertilidade de solos do Amapá. Semina. Ciências Agrárias, v.29, p.499-506, 2008.

MOHEBODINI, M.; DEHGHANI, H.; SABAGHPOUR, S.H. Stability of performance in lentil (Lens culinaris Medik) genotypes in Iran. Euphytica, v.149, p.343-352, 2006.

PELÚZIO, J.M.; FIDELIS, R.R.; GIONGO, P.; SILVA, J.C. da; CAPPELLARI, D.; BARROS, H.B. Adaptabilidade e estabilidade de cultivares de soja em quatro épocas de semeadura no sul do Estado do Tocantins. Revista Ceres, v.55, p.34-40, 2008.

PLAISTED, R.L.; PETERSON, L.C. A technique for evaluating the ability of selection to yield consistently in different locations or seasons. American Potato Journal, v.36, p.381-385, 1959.

ROCHA, R.B.; MURO-ABAD, J.I.; ARAUJO, E.F.; CRUZ, C.D. Avaliação do método centroide para estudo de adaptabilidade ao ambiente de clones de Eucalyptus grandis. Ciência Florestal, v.15, p.255-266, 2005.

SILVA, W.C.J. e; DUARTE, J.B. Métodos estatísticos para estudo de adaptabilidade e estabilidade fenotípica em soja. Pesquisa Agropecuária Brasileira, v.41, p.23-30, 2006.

VASCONCELOS, E.S. de; BARIONI JÚNIOR, W.; CRUZ, C.D.; FERREIRA, R. de P.; RASSINI, J.B.; VILELA, D. Seleção de genótipos de alfafa pela adaptabilidade e estabilidade da produção de matéria seca. Acta Scientiarum. Agronomy, v.30, p.339-343, 2008.

Recebido em 6 de outubro de 2008 e aprovado em 27 de fevereiro de 2009 